\title{
Anabases
}

ANABASES Traditions et réceptions de l'Antiquité

$14 \mid 2011$

Varia

\section{Scènes de latrines dans le roman historique, le cinéma et la bande dessinée}

Alain Bouet

\section{OpenEdition}

1 Journals

Édition électronique

URL : http://journals.openedition.org/anabases/2344

DOI : 10.4000/anabases.2344

ISSN : 2256-9421

Éditeur

E.R.A.S.M.E.

Édition imprimée

Date de publication : 1 octobre 2011

Pagination : 221-230

ISSN : 1774-4296

\section{Référence électronique}

Alain Bouet, « Scènes de latrines dans le roman historique, le cinéma et la bande dessinée », Anabases [En ligne], 14 | 2011, mis en ligne le 01 octobre 2014, consulté le 20 octobre 2019. URL : http:// journals.openedition.org/anabases/2344; DOI : 10.4000/anabases.2344

Ce document a été généré automatiquement le 20 octobre 2019.

(c) Anabases 


\title{
Scènes de latrines dans le roman historique, le cinéma et la bande dessinée
}

\author{
Alain Bouet
}

Nous tenons à remercier M. Feugère et Fr. Cadiou pour leurs conseils.

Gladiator a tout changé. Le film de Ridley Scott sorti en 2000, par le succès rencontré, a non seulement relancé la mode du peplum, mais présente une vision différente de la ville antique et de Rome en particulier : une ville violente et crasseuse. Par l'image qu'elles véhiculent dans la conscience collective, les latrines antiques appartiennent à cette sphère (fig. 1). Ben Hur n'allait pas aux latrines ${ }^{16}$; Maximus, le héros de Gladiator, aurait pu, en revanche, les fréquenter. Ce n'est pas un hasard si la première scène de latrines - ou une des toutes premières - du roman historique apparaît dans le sulfureux, transgressif et remarquablement documenté Neropolis d'H. Monteilhet ${ }^{17}$. Le même constat pourrait être dressé pour la bande dessinée : la série Murena de Dufaux et Delaby ${ }^{18}$ compte également sa scène de latrines. Même le propret Alix fréquente désormais ces lieux ${ }^{19}$ qui font maintenant partie intégrante des ressorts scénaristiques. 
Fig. 1. Les latrines des Thermes des Cyclopes à Dougga : vocabulaire inhérent à ces installations (cl. A. Bouet).

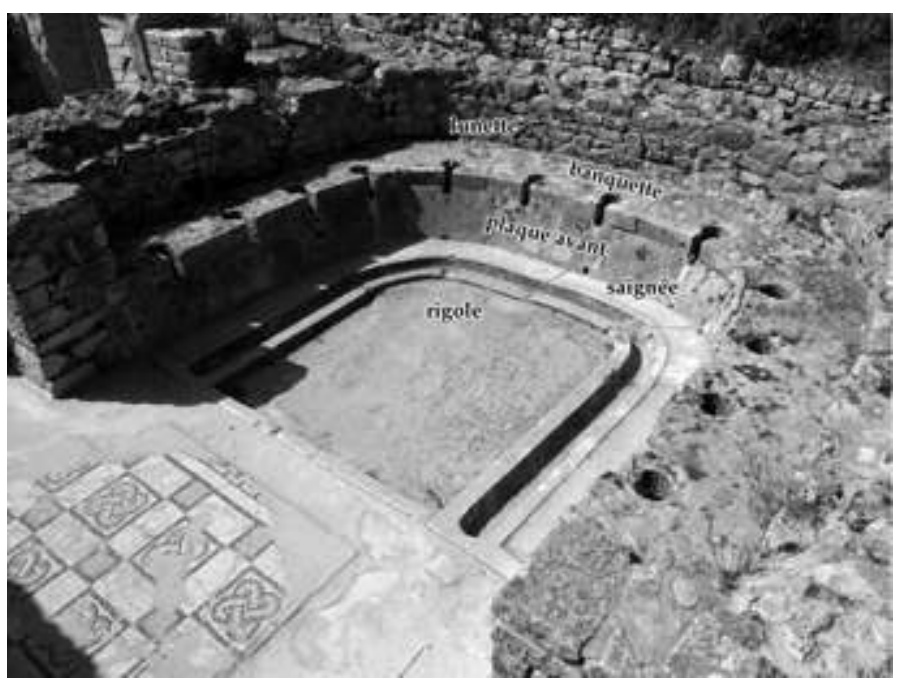

\section{Description des latrines}

2 H. Monteilhet consacre plus d'une page ${ }^{20}$ à la description de latrines fictives qu'il situe au Forum et que fréquente son héros, Kaeso. Ces latrines en hémicycle de vingt-quatre places, chauffées par hypocauste, richement décorées, sont précédées d'un vestiaire dans lequel le citoyen peut ôter sa toge. Les sièges sont séparés par des accoudoirs sculptés en forme de dauphins. Au-dessus, trois niches accueillent les statues de Fortuna, Esculape et Bacchus. Elles font face à sept autres dans lesquelles trônent les bustes des Sept Sages. Les lunettes sont décrites ainsi que les éponges (venues d'Afrique ou de Grèce) servant à s'essuyer après avoir été trempées dans une rigole alimentée en eau propre située au-devant des sièges. Le centre de la pièce est occupé par une vasque servant de lavabo. L'ambiance du lieu est également évoquée : lieu de rendez-vous ou de rencontres pour les homosexuels. L'auteur cite alors l'épigramme de Martial sur Vacerra $^{21}$. Après cette description visuelle, suit une évocation olfactive : «Et l'homme qui apprécie les senteurs de son propre étron bien moulé, apprécie beaucoup moins d'ordinaire les étrons des autres ${ }^{22}$.»

Ce sont les latrines du palais que fréquente l'empereur Claude au début du sixième épisode de la série Moi Claude empereur ${ }^{23}$. Elles comptent au moins deux places, séparées par des accoudoirs ornés de dauphins. La lunette ne possède aucune saignée verticale sur la plaque avant. Le sol est décoré d'une mosaïque noire et blanche.

Dans plusieurs films ou bandes dessinées, les latrines présentent certains points communs. Dans l'épisode 3 de la saison 2 de la série Rome $e^{24}$, une scène particulièrement forte s'y déroule. La banquette court au moins sur deux côtés d'une pièce située en contrebas par rapport à la rue. On y descend par l'intermédiaire d'un escalier de quatre marches. Les lunettes possèdent une courte saignée qui se prolonge sur la plaque avant. La banquette est haute puisque les pieds des usagers ne touchent pas le sol. Des éponges sont rassemblées dans un seau en bois. La pièce est ouverte sur la rue par l'intermédiaire de larges baies simplement obturées par une balustrade basse.

Dans le premier épisode ${ }^{25}$ de la saison 2 de Spartacus, Gods of Arena, le laniste Batiatus et son ami Solonius vont aux latrines, non loin de l'amphithéâtre en construction de 
Capoue. Celles-ci, relativement modestes (minimum 6 places), sont largement ouvertes et en surélévation d'une marche sur la rue. Un poteau de bois au moins soutient une poutre horizontale supportant le premier étage du bâtiment dans lequel elles sont aménagées. Dans la largeur des ouvertures, sont fixés des rideaux roulés en position ouverte dont on ne comprend guère l'utilité. Certains usagers sont assis pour déféquer, d'autres, debout, urinent au travers des lunettes. De celles-ci, on ne distingue que la saignée verticale qui se prolonge sur la plaque avant. Batiatus se nettoie avec une éponge fixée au bout d'un manche, fournie par un esclave noir, probable gardien du lieu. On note l'absence d'eau - à moins qu'elle ne se trouve dans le seau où sont entreposées les éponges -, ce qui ne devait pas faciliter l'opération.

Une courte scène du remarquable film Agora ${ }^{26}$ présente des latrines en $U$ d'une dizaine de places située dans l'enceinte du Serapeum et de la bibliothèque d'Alexandrie. Les lunettes ont un large diamètre, comme l'ouverture circulaire de la plaque avant. Des éponges sont entreposées dans un seau. La rigole est ici remplacée par un grand bassin quadrangulaire peu profond à l'eau marronnâtre. Les parois sont constituées de panneaux à claire-voie disposés entre des colonnes égyptiennes.

Dans Brûlez Rome $e^{27}$ !, la reconstitution des latrines se veut plus authentique puisqu'il s'agit d'un docu-fiction. Elles sont aménagées au sous-sol d'une insula. Un puissant pilier au centre de la salle soutient l'étage. Une banquette court vraisemblablement sur trois côtés de la pièce et doit compter plus de dix places. Les lunettes ont un diamètre normal. La banquette n'est pas uniforme, mais possède - toutes les places ou les deux places - une sorte de petit accoudoir. Elle est dotée d'un dossier qui fait ressembler l'ensemble à un large fauteuil. Cette banquette présente des hauteurs différentes selon les tronçons. Dans certains, les pieds des usagers ne touchent pas le sol. L'ensemble repose sur un puissant marchepied. Il y a absence de rigole. Au centre d'une des parois, la banquette est remplacée par une fontaine dont la borne est ornée d'une divinité masculine (?) en buste, non identifiable. Un seau (ou plus) contient des éponges et leurs bâtons, tandis qu'un balai est appuyé contre le pilier central.

Tous ces exemples présentent des salles fortement délabrées ou crasseuses. L'ambiance est différente chez Alix. Dans Le testament de César ${ }^{28}$, une poursuite se déroule dans des latrines rattachées à une taverne, désignée comme un des lieux les plus mal famés de Rome. La pièce est simplement séparée du reste de la construction par une sorte de paillasse sur laquelle est déposé un bol contenant des éponges. Les murs sont en opus mixtum, reticulatum et briques. Une fenêtre haute, sans vitre, éclaire la salle. La banquette, disposée en $U$, est percée d'une dizaine de lunettes. Elles comprennent, dans la plaque avant, une large ouverture identique à la lunette. Le sol dallé est creusé d'une rigole parallèle à la banquette.

9 La dernière parution de la série Les voyages d'Alix reconstitue la Vienne antique. Sont présentées les latrines des Thermes des Lutteurs de Saint-Romain-en-Gal ${ }^{29}$. On y retrouve une pièce trapézoïdale avec une banquette percée sur trois côtés faisant face à une fontaine monumentale.

Dans le tome III de La dernière prophétie, les latrines n'apparaissent que furtivement au travers d'une vignette ${ }^{30}$. La banquette disposée en $\mathrm{U}$ comporte des lunettes, ainsi qu'un aménagement dans la plaque avant identique à celui représenté dans l'album d'Alix précédemment évoqué. La base des parois est lambrissée, le sol recouvert d'une mosaïque à écailles imbriquées noires et blanches. L'impression de luxe est bien rendue dans ces lieux probablement situés dans le palais impérial au début du III siècle ap. J.-C. 
11 Une scène du tome 1 de la série Arelate se déroule également dans des latrines ${ }^{31}$. Le cadre est celui de la ville d'Arles à l'époque flavienne au moment de la construction de l'amphithéâtre. Les lieux se situent dans l'enceinte des thermes dits de l'Esplanade fouillés entre 1974 et 2000. On y accède directement depuis une des salles du bain dotée d'une vaste piscine centrale qui trouverait plus sa place dans des thermes thérapeutiques qu'hygiéniques. La baie n'est pas obturée par une porte ou une tenture. Les lunettes de la banquette ont un diamètre important et sont dotées, sur la plaque avant, d'un aménagement identique à celui d'Ostie.

12 La bande dessinée Murena ${ }^{32}$ met en avant des latrines situées près du Portique de Pompée. La pièce se termine à une de ses extrémités par une exèdre. Les parois sont recouvertes d'une peinture agrémentée de graffiti relatifs aux combats de gladiateurs et dans une moindre mesure à la sexualité. Les lunettes de la banquette présentent un large diamètre. Au-devant, la rigole est parcourue par un flux d'eau dans lequel trempent quelques éponges fixées au bout de bâtons. D'autres sont disposées dans un bassin monolithe situé plus avant. La pièce est largement ouverte sur la rue par une colonnade ionique.

\section{Respect de la réalité archéologique ou liberté d'interprétation?}

La restitution des latrines présente pour la plupart d'entre elles des approximations. Le lecteur reconnaîtra dans la description flamboyante des latrines de Neropolis celle faite par J. Carcopino dans sa Vie quotidienne à Rome à l'apogée de l'Empire ${ }^{33}$. Dans ces pages, l'auteur présente la documentation alors connue, à savoir les latrines de Rome, de Pompéi, de Timgad et d'Ostie dont les latrines de Monteilhet constituent un mélange. La forme courbe de la pièce reprend celle des installations sanitaires du Forum de César mises en place à l'époque de Trajan (et donc inexistantes à celle de Néron); il en va de même pour le chauffage : on sait, depuis 1991, que si la pièce est bien dotée de pilettes, il ne s'agit pas pour autant d'un hypocauste. Cet aménagement est lié à l'emplacement particulier de la salle, accessible par le Clivus Argentarius mais située au premier étage par rapport au niveau de circulation de la place. Afin de ne pas alourdir inutilement le poids de la maçonnerie sur les substructures, les architectes ont choisi, pour créer une différence de niveau suffisante leur permettant d'installer un égout, des pilettes maintenant un sol suspendu plutôt que des remblais ${ }^{34}$. La statue de Fortuna s'inspire de celle qui devait orner les latrines de la Caserne des Vigiles à Ostie (II, V, ${ }^{35}$ ). Quant aux Sept Sages, ils doivent être rapprochés de leur représentation dans l'apodyterium des thermes éponymes d'Ostie (III, X, 2). Sur les parois, les sentences qui leur sont attribuées ont un sens scatologique, mais on pourrait aussi évoquer le décor des latrines de la maison II d'Éphèse également pourvues de représentations de personnages interprétés comme des philosophes ${ }^{36}$. Quant aux accoudoirs en forme de dauphins, ils se rattachent directement aux toilettes situées près du forum à Timgad où ils servent d'éléments séparant une ou deux places. Toutefois, au vu des clichés publiés, nous pensons que ce décor est le fait d'une restitution erronée à laquelle il faut substituer une banquette percée continue, ornée vraisemblablement à ses extrémités par de tels accoudoirs. Ceux-ci existent toutefois dans les latrines de la Domus Augustana à Rome. Mais ce sont là des structures très particulières, constituées de trois niches accueillant chacune une place et probablement liées au cercle impérial ${ }^{37}$. De tels 
accoudoirs sont d'ailleurs présents dans les latrines du palais de la série Moi, Claude empereur.

Dans la série Rome, si les lunettes paraissent correctement représentées, la banquette est toutefois trop haute car les pieds des usagers ne touchent pas le sol. Il en est de même dans le docu-fiction Brûlez Rome!

Par leur forme et leur architecture, les latrines de l'album d'Alix sont directement inspirées de celles de la voie de la Forica (I XII, 1) à Ostie, datées de la fin du IV siècle ap. J.-C. La fenêtre qui laisse entrer une abondante lumière ne présente aucune similitude avec les aménagements du début de la seconde moitié du $\mathrm{I}^{\mathrm{er}}$ siècle av. J.-C., époque à laquelle est censée se dérouler l'histoire. L'absence d'enduit, même modeste, sur les parements, de vitrage à la fenêtre, d'eau coulant dans la rigole au-devant de la banquette donne l'impression que la scène se déroule dans des vestiges archéologiques. Dans la série des Voyages d'Alix, nous ne sommes plus dans le domaine de la fiction qui autorise toutes les approximations, mais dans celui de la reconstitution historique la plus précise telle que revendiquée en début de volume. Le résultat est pour le moins déplorable, malgré des vestiges remarquablement conservés. Les lunettes de la banquette percée ont un diamètre bien trop important, le trop-plein de la fontaine qui fait face aux usagers, pourtant retrouvé, n'est même pas représenté, comme le décor de scène de lutte stylisé à l'extrême, en partie mis au jour dans le comblement de l'égout. Et pourtant, la découverte en est remarquable...

Dans l'album La dernière prophétie précédemment évoqué, la base des parois est lambrissée, le sol recouvert d'une mosaïque à écailles imbriquées noires et blanches qui n'est pas sans rappeler le sol des latrines des thermes des Filadelfes à Timgad ${ }^{38}$.

Dans la série Arelate, les thermes dits de l'Esplanade ont été reconstitués bien plus vastes qu'ils ne l'étaient en réalité et pourvus de latrines qui n'existaient pas. L'auteur les a rajoutées pour servir l'action. Leur ouverture sur une des pièces du bain n'est pas crédible. Si cette salle se retrouve fréquemment dans l'environnement thermal, c'est qu'elle profite, pour nettoyer l'égout latéral, de l'abondance de l'eau usée provenant de la vidange des bassins. Néanmoins, aucune n'ouvre directement sur une des pièces balnéaires.

Plus ambitieuse et précise est la reconstitution des latrines de la série Murena. Le lecteur reconnaîtra aisément la Grande Forica du Largo Argentina. La pièce est précisément reconstituée avec son exèdre terminale et son décor identique à celui archéologiquement attesté. Ici aussi, les lunettes de la banquette apparaissent surdimensionnées par rapport à quelques-unes d'entre elles retrouvées en fouille ${ }^{39}$. Si la rigole est attestée par l'archéologie, en revanche tel n'est pas le cas du bassin monolithe situé plus avant. Ce dernier n'est pas sans rappeler celui mis au jour dans les latrines $\mathrm{du}$ fort de Housesteads à Caerlaverock le long du mur d'Hadrien. L'emplacement des supports des colonnes a bien été retrouvé en limite de fouille, mais c'est leur interprétation qui est ici erronée. Il ne s'agit pas d'une colonnade ouvrant sur une voie, mais d'une colonnade intérieure à la pièce, à l'instar de celle des latrines découvertes à Rottembourg am Neckar (Allemagne) ${ }^{40}$. Ce secteur du portique de Pompée est relativement bien connu, car aux vestiges archéologiques s'ajoutent les informations de la forma Urbis. Or il n'existe pas de rue à cet emplacement. Ces latrines devaient ouvrir sur l'aire centrale du complexe par l'intermédiaire d'une simple porte. 
Transparaît ici l'idée généralement admise que les Romains faisaient leurs besoins en public. Or ceci est totalement erroné. L'ensemble des vestiges archéologiques montre au contraire que les latrines formaient un lieu clos, refermé sur lui-même, dans lequel un certain nombre d'aménagements avait été prévu - rideau, entrée en chicane... -, pour éviter que tout regard extérieur puisse se perdre dans la pièce. Ceci garantissait donc une intimité collective. Le lieu rassemblait des personnes réunies dans un même but, ce qui est très différent de l'idée de personnes déféquant en public. Le type vestimentaire - la tunique - comme la pénombre qui devait au moins régner dans les latrines les plus anciennes constituaient autant de facteurs qui ne devaient guère permettre à un Romain de jauger les attributs de ses contemporains. D'ailleurs, à elle seule, la vision des parties génitales ne devait pas gêner dans une société où l'activité sportive et la baignade se pratiquaient nu. Ce qui devait embarrasser, c'était plutôt l'attitude dépourvue de dignité de celui qui se trouvait aux latrines: le fait d'être accroupi, le visage peut-être marqué par l'effort ou crispé. Dès lors, on comprend mieux la saillie de Suétone (Vespasien, 20) rapportant les railleries proférées à l'encontre de Vespasien au visage toujours crispé, comme s'il était en train de déféquer ${ }^{43}$. Seules trois latrines étudiées ici retranscrivent cette nette séparation entre intérieur et extérieur : celles de Neropolis dans lesquelles un vestiaire est mentionné, celles de Brûlez Rome! situées en sous-sol, et celles de Moi Claude empereur fermées par une porte.

21 L'essuyage avec éponge fixée au bout d'un bâton tel que connue par les sources antiques est représenté la plupart du temps ${ }^{44}$. À noter toutefois de grandes différences dans la longueur des bâtons - avec un surdimensionnement évident dans la série Rome et dans le docu-fiction Brûlez Rome! - dont la cause tient probablement au désir du réalisateur d'en faire un élément clairement identifiable par le spectateur. La rigole d'eau claire n'est pour sa part pas systématiquement figurée ; elle est parfois remplacée par un simple seau ou un grand bassin. L'existence de fontaines n'est attestée que deux fois, dans le roman Neropolis et dans le docu-fiction Brûlez Rome! Celles-ci sont cependant, à plusieurs reprises, attestées par l'archéologie ${ }^{45}$.

Deux scènes seulement, dans la bande dessinée Murena et le docu-fiction Brûlez Rome!, abordent le problème de l'éclairage des latrines publiques qui devaient être accessibles la nuit. Dans celles du portique de Pompée, des torches sont fichées au mur alors que pendent au plafond de curieuses lampes à huile dont on ne retrouve pas de comparaisons antiques et dont le modèle doit être recherché dans les lustres des années 1920 ! Dans celles du docu-fiction, une série de lustres à une dizaine de becs est suspendue au plafond. Si l'archéologie ne fournit aucune indication pour l'éclairage des latrines publiques, l'usage de torches paraît toutefois peu probable. On note simplement, dans quelques latrines privées de Pompéi, la présence de modestes niches ou de consoles destinées à poser une lampe à huile ${ }^{46}$. Dans la série Moi Claude empereur, Claude se rend aux lieux de nuit ; il s'éclaire grâce à une lampe à huile en bronze à deux becs qu'il pose sur le siège voisin du sien. 


\section{Dans les latrines, évocation de scènes de la vie quotidienne?}

L'endroit très particulier que sont les latrines est utilisé par les auteurs et les scénaristes comme cadre de scènes très diverses.

Dans Agora, il s'agit d'une brève vision servant à illustrer la routine de vie qui s'est installée à l'intérieur du Serapeum et de la bibliothèque d'Alexandrie, assiégés par les chrétiens. Dans Spartacus, il est également question d'une simple scène de vie quotidienne. Celle décrite dans Brûlez Rome ! est du même ordre, même si l'action y est plus importante. L'instructeur Similis et ses vigiles reçoivent un seau d'urine jeté depuis les étages par un habitant. Celui-ci s'en défend, indiquant qu'il revient des latrines voisines; le malheureux y est traîné pour vérification. Cette scène aurait pu être l'occasion de filmer, à la place d'un simple seau de bois, une partie du riche vaisselier de nuit connu des Romains : lasanum - pot de chambre - ou matella - urinoir masculin ${ }^{47}$. Cette vaisselle n'est présentée que dans la série Moi Claude empereur; l'empereur vient se soulager aux latrines en apportant un vase rempli d'urine qu'il vide par la lunette. Celui-ci ressemble plus à un calice qu'à une matella qui, d'après les descriptions antiques, correspond à une forme céramique fermée proche d'une cruche.

Quant au héros de Neropolis, Kaeso, il se rend aux latrines du forum pour y écouter prêcher les premiers disciples du Christ que sont Luc et Paul. Dans la série Moi Claude empereur, le souverain s'y assoupit, laissant libre cours à ses souvenirs et ne se réveille qu'en fin d'épisode.

Dans bon nombre de cas toutefois, il s'agit de scènes violentes. Dans La dernière prophétie, c'est l'assassinat d'Héliogabale dans des latrines tel que rapporté dans l' Histoire Auguste ${ }^{48}$ qui est représenté. Dans Le testament de César, Alix poursuit un malfaiteur qui tente de s'échapper par la fenêtre haute de la pièce. Dans Murena comme dans Rome, les séquences sont identiques. Dans la bande dessinée, le malheureux Silvanus va finir sa vie la tête dans la lunette, asphyxié par ses excréments. Dans Rome, l'ultime rebondissement de la vengeance entre habitants du Caelius et de l'Aventin se déroule dans les latrines: Carbo, un des hommes de main du Caelius, est sodomisé, la tête dans une lunette.

Ces scènes sont invraisemblables. Dans la première, Silvanus ne peut périr ainsi parce que les excréments tombent dans l'égout situé au-dessous de la banquette et sont évacués au loin. Ils ne sont à aucun moment stockés sur place ${ }^{49}$. D'autre part, le diamètre des lunettes antiques est trop modeste pour laisser passer une tête adulte. À quelques détails près, il est étonnant de voir la même séquence retranscrite, à des dates voisines, sur l'écran comme sur le papier. Elles ne sont pas d'ailleurs sans rappeler certains polars ${ }^{50}$ et un effet de mode.

Dans les latrines d'Arelate, Vitalis se fait également rouer de coup et menacer par un couteau.

Dans quelques autres cas, les latrines ne sont qu'évoquées. Ainsi, à Rome, dans le roman Ce soir on dîne chez Pétrone, Pétrone passe en litière devant la curie de Pompée transformée en latrines publiques ${ }^{51}$, évocation rapide de l'épisode rapporté par Dion Cassius suite à l'assassinat de César (XLVII, 19) ${ }^{52}$. Dans le film Centurion ${ }^{53}$, est simplement cité par les soldats de T. Flavius Virilus, général de la IX ${ }^{e}$ légion, un long 
graffito à sa gloire gravé dans les latrines : «À l'entraînement il est notre maître, Au banquet notre pair, Dans la troupe notre frère, Et au combat il est le dieu sur qui nous comptons pour sauver nos âmes. » Ce type de texte n'a évidemment pas été découvert dans de tels endroits; les graffiti ont trait plutôt à l'expression de la satisfaction des besoins naturels alors que les inscriptions à caractère sexuel sont très rares ${ }^{54}$. Dans L'aigle de la Neuvième Légion ${ }^{55}$, le centurion par intérim L. Drusilius Salinator signale à son successeur M. Flavius Aquila qui vient prendre possession du fort qu'un de ses hommes s'emploie à régler un problème avec les latrines et qu'il y travaille d'ailleurs sans trop de succès. Il est peu probable qu'il s'agisse de la vidange d'une latrine sur fosse car cela ne relèverait pas d'une difficulté occasionnelle, mais d'un entretien normal. Il est plus probablement question du débouchage d'un égout. Cette remarque furtive destinée à rendre plus palpable la vie quotidienne d'un camp rappelle que du personnel était attaché à l'entretien de ces endroits, tel M. Longinus A[---], soldat de la legio III Cyrenaica, qui, la veille des nones d'octobre 90 ap. J.-C. était de corvée de stercus $^{56}$.

\section{Conclusion}

Les latrines sont donc utilisées par les scénaristes ou les auteurs parce qu'elles sont perçues comme des endroits sordides, propices à des déchaînements de violence ou comme des lieux transgressifs marquant l'esprit et l'imagination du spectateur ou du lecteur. Elles sont bien éloignées de la réalité archéologique qui, si elle était respectée, serait bien moins spectaculaire. Dans ce panorama décevant, surnagent toutefois la description d'H. Monteilhet et la représentation de Moi Claude empereur, les premiers à évoquer précisément par le texte et l'image ces structures anecdotiques adaptées pour l'occasion à leur propos. Aussi, bien que ces récits de fiction soient empreints de quelques inexactitudes, l'action qui se déroule dans ces lieux, tout à fait originale (et crédible pourquoi pas!), en fait des exemples intéressants...

\section{BIBLIOGRAPHIE}

BOUCHARD, G. ; HELly, Br. et MARTIN, J. Les voyages d'Alix, Vienna, Casterman, 2011.

BOUET, A. Les latrines dans les provinces gauloises, germaniques et alpines, Gallia, suppl. 59, Paris, 2009.

CARCOPINO, J. La vie quotidienne à Rome à l'apogée de l'Empire, Paris, 1939.

CHAILLET, G. La dernière prophétie, III. Sous le signe de Ba'al, Glénat, 2004.

COMBescot, P. Ce soir on dîne chez Pétrone, Grasset, 2004.

DUFAUX, DELABY. Murena, t. 6, Le sang des bêtes, Dargaud, 2007.

D’HAucourt, A. « Peinture ou théâtre ? Louis Feuillade, Héliogabale et le cinéma français en 1911 », Journal of Inquiry and Research 84/2006, 107-123. 
MARTIN, J. ; VENANZI, M. Le testament de César, Casterman, 2010.

MONTEILHET, H. Neropolis, roman des temps néroniens, Paris, 1984.

SIEURAC, L. ; GENOT, A. Arelate, t. 1. Vitalis, Idées, 2009.

\section{NOTES}

16. En 1911, L. Feuillade tourne un court métrage de 8' 33" intitulé Héliogabale, connu également sous le titre L'Orgie romaine. Plusieurs scènes s'inspirent de faits rapportés dans l'Histoire Auguste. Si le film est déjà très audacieux en représentant l'assassinat de l'empereur, celui-ci ne se déroule toutefois pas dans les latrines comme évoqué dans la source antique (Héliogabale, XVII, 1), mais dans une pièce anonyme; il existait chez Gaumont une série de règles à respecter et il est possible que les latrines aient été jugées comme trop scabreuses (D’Hautcourt 2006, p. 111).

17. Monteilhet 1984.

18. Dufaux, Delaby 2007.

19. Martin, Venanzi 2010.

20. Monteilhet 1984, p. 310-312.

21. «Il a envie de dîner et non pas de chier, ce Vacerra qui passe ses heures et siège des journées entières dans tous les chalets de nécessité » (Épigrammes, XII, 77).

22. Monteilhet 1984, p. 312.

23. Une certaine justice. Feuilleton télévisé britannique en 13 épisodes créé par J. Pulman, sorti en 1976 (00' 33" à 02' 15 " et 49' 50" à 50' 25").

24. Le message de Cicéron. Série télévisée américano-britannico-italienne créée par J. Milius, W. MacDonald et Br. Heller. Saison 1 sortie en 2005, saison 2 en 2007 (23' 51" à 24' 35").

25. Épisode 1 intitulé Past Transgressions. Série américaine créée par St. S. Denight, 2011 (10'44" à 11' 45").

26. Film hispano-maltais de A. Amenábar, 2009 (37' 20" à 37' 25").

27. Docu-fiction français de R. Kéchichian, 2005 (29' 37" à 29' 42").

28. Martin, Venanzi 2010, p. 38.

29. Bouchard et al. 2011, p. 30.

30. Chaillet 2004, p. 47, vignette 2.

31. Sieurac, Genot 2004, p. 44-47.

32. Dufaux, Delaby 2007, p. 2-4.

33. Carcopino 1939, p. 58-60.

34. Bouet 2009, p. 136-137.

35. Ibid., p. 144.

36. Ibid., p. 143.

37. Ibid., p. 65.

38. Ibid., p. 139.

39. Elles mesurent $14,8 \mathrm{~cm}$ et $16 \mathrm{~cm}$ de diamètre.

40. Bouet 2009, p. 50-51.

41. Ibid., p. 102. Elles sont représentées avec un diamètre correct dans la série Moi Claude empereur.

42. Bouet 2009, p. 101.

43. Ibid., p. 174-175.

44. Ibid., p. 172.

45. Ibid., p. 132-133.

46. Ibid., p. 175. 
47. Ibid., p. 64-75.

48. Voir note 1 .

49. Ceci n'empêche pas que des odeurs d'excréments puissent flotter dans l'air de la pièce comme évoqué dans Neropolis.

50. Par exemple, The Big Lebowski, film américain de J. et E. Coen, 1998; The Untold Story, film hongkongais de $\mathrm{H}$. Yau, 1993.

51. Combescot 2004, p. 140 ( Probablement, un jour, les excréments de la plèbe embreneront nos plus héroïques souvenirs, puisque l'honnête citoyen chie et pisse là même où, jadis, le grand César fut proprement suriné »).

52. L'archéologie a montré que la curie n'a pas été transformée directement en latrines, mais que des latrines ont été aménagées à proximité immédiate. Il s'agit de la Grande Forica précédemment évoquée et dessinée dans Murena.

53. Film britannique de N. Marshall, 2010 (20' 30").

54. Bouet 2009, p. 180.

55. Film britannico-américain de K. MacDonald, 2011 (3' 58").

56. $P$. Gen. Lat. 1 recto $V=C P L, 106$. On ne peut toutefois pas trancher entre la vidange des latrines et le nettoyage du fumier (Bouet 2009, p. 169).

\section{AUTEUR}

\section{ALAIN BOUET}

Université de Toulouse (UTM), TRACES

abouet@univ-tlse2.fr 